\title{
Primary Prevention of Cardiovascular Disease with a Mediterranean Diet Supplemented with Extra-Virgin Olive Oil or Nuts
}

\author{
R. Estruch, E. Ros, J. Salas-Salvadó, M.-I. Covas, D. Corella, F. Arós, \\ E. Gómez-Gracia, V. Ruiz-Gutiérrez, M. Fiol, J. Lapetra, R.M. Lamuela-Raventos, \\ L. Serra-Majem, X. Pintó, J. Basora, M.A. Muñoz, J.V. Sorlí, J.A. Martínez, M. Fitó, \\ A. Gea, M.A. Hernán, and M.A. Martínez-González, \\ for the PREDIMED Study Investigators*
}

ABSTRACT

\section{BACKGROUND}

Observational cohort studies and a secondary prevention trial have shown inverse associations between adherence to the Mediterranean diet and cardiovascular risk.

\section{METHODS}

In a multicenter trial in Spain, we assigned 7447 participants ( 55 to 80 years of age, 57\% women) who were at high cardiovascular risk, but with no cardiovascular disease at enrollment, to one of three diets: a Mediterranean diet supplemented with extra-virgin olive oil, a Mediterranean diet supplemented with mixed nuts, or a control diet (advice to reduce dietary fat). Participants received quarterly educational sessions and, depending on group assignment, free provision of extra-virgin olive oil, mixed nuts, or small nonfood gifts. The primary end point was a major cardiovascular event (myocardial infarction, stroke, or death from cardiovascular causes). After a median follow-up of 4.8 years, the trial was stopped on the basis of a prespecified interim analysis. In 2013, we reported the results for the primary end point in the Journal. We subsequently identified protocol deviations, including enrollment of household members without randomization, assignment to a study group without randomization of some participants at 1 of 11 study sites, and apparent inconsistent use of randomization tables at another site. We have withdrawn our previously published report and now report revised effect estimates based on analyses that do not rely exclusively on the assumption that all the participants were randomly assigned.

RESULTS

A primary end-point event occurred in 288 participants; there were 96 events in the group assigned to a Mediterranean diet with extra-virgin olive oil (3.8\%), 83 in the group assigned to a Mediterranean diet with nuts (3.4\%), and 109 in the control group (4.4\%). In the intention-to-treat analysis including all the participants and adjusting for baseline characteristics and propensity scores, the hazard ratio was 0.69 (95\% confidence interval [CI], 0.53 to 0.91$)$ for a Mediterranean diet with extra-virgin olive oil and $0.72(95 \% \mathrm{CI}$, 0.54 to 0.95 ) for a Mediterranean diet with nuts, as compared with the control diet. Results were similar after the omission of 1588 participants whose study-group assignments were known or suspected to have departed from the protocol.

CONCLUSIONS

In this study involving persons at high cardiovascular risk, the incidence of major cardiovascular events was lower among those assigned to a Mediterranean diet supplemented with extra-virgin olive oil or nuts than among those assigned to a reduced-fat diet. (Funded by Instituto de Salud Carlos III, Spanish Ministry of Health, and others; Current Controlled Trials number, ISRCTN35739639.)
The authors' full names, academic degrees, and affiliations are listed in the Appendix. Address reprint requests to Dr. Martínez-González at the Department of Preventive Medicine and Public Health, Facultad de Medicina-Clínica Universidad de Navarra, Irunlarrea 1, 31008 Pamplona, Spain,or at mamartinez@unav.es.

*The PREDIMED study investigators are listed in the Supplementary Appendix, available at NEJM.org.

Drs. Estruch and Martínez-González contributed equally to this article.

This article was published on June 13,2018 , at NEJM.org.

N Engl J Med 2018;378:e34. DOI: 10.1056/NEJMoa1800389

Copyright @ 2018 Massachusetts Medical Society. 
T HE TRADITIONAL MEDITERRANEAN DIET is characterized by a high intake of olive oil, fruit, nuts, vegetables, and cereals; a moderate intake of fish and poultry; a low intake of dairy products, red meat, processed meats, and sweets; and wine in moderation, consumed with meals. ${ }^{1}$ In observational cohort studies ${ }^{2,3}$ and a secondary prevention trial (the Lyon Diet Heart Study), ${ }^{4}$ increasing adherence to the Mediterranean diet has been consistently associated with lower cardiovascular risk..$^{2-4} \mathrm{~A}$ systematic review ranked the Mediterranean diet as the most likely dietary model to provide protection against coronary heart disease. ${ }^{5}$ Small clinical trials have uncovered plausible biologic mechanisms to explain the salutary effects of this food pattern. ${ }^{6-9}$ We designed a randomized trial, PREDIMED (Prevención con Dieta Mediterránea), to test the efficacy of two Mediterranean diets (one supplemented with extra-virgin olive oil and another with nuts), as compared with a control diet (advice on a low-fat diet), on primary cardiovascular prevention. Our original report was published in the Journal in 2013. ${ }^{10}$ A 2017 analysis $^{11}$ of the distributions of baseline variables in randomized trials identified the PREDIMED trial as having distributions that were significantly different from what would have been expected to result from randomization. This report led to our conducting a review of how participants were assigned to various intervention groups; that review revealed irregularities in our randomization procedures. Therefore, we have withdrawn our original report ${ }^{12}$ and now publish a new report.

We describe the protocol deviations and report revised intention-to-treat and per-protocol effect estimates that do not rely exclusively on the assumption that all the participants had been randomly assigned to the intervention groups. A detailed description of the changes that have been introduced and departures from the protocol is provided in the Supplementary Appendix, available with the full text of this article at NEJM.org.

\section{METHODS}

\section{STUDY DESIGN}

The PREDIMED study was designed as a parallelgroup, multicenter, randomized trial. Details of the study design have been reported previously. ${ }^{13,14}$ The protocol, available at NEJM.org, was approved by the institutional review boards at all study locations. The authors vouch for the accuracy and completeness of the data and all analyses and attest that this report accurately describes the conduct of the study as we know it.

Funding was provided by grants from Instituto de Salud Carlos III, Spanish Ministry of Health. Supplemental foods were donated, including extra-virgin olive oil (by Hojiblanca and Patrimonio Comunal Olivarero, both in Spain), walnuts (by the California Walnut Commission), almonds (by Borges, in Spain), and hazelnuts (by Morella Nuts, in Spain). None of the sponsors had any role in the study design, data analysis, or reporting of the results.

\section{PARTICIPANT SELECTION AND RANDOMIZATION}

Eligible participants were men (55 to 80 years of age) or women (60 to 80 years of age) with no cardiovascular disease at enrollment, who had either type 2 diabetes mellitus or at least three of the following major risk factors: smoking, hypertension, elevated low-density lipoprotein cholesterol levels, low high-density lipoprotein cholesterol levels, overweight or obesity, or a family history of premature coronary heart disease. Detailed enrollment criteria are provided on pages 18 and 19 in the Supplementary Appendix. All the participants provided written informed consent.

The protocol specified that participants were to be randomly assigned, in a 1:1:1 ratio, to one of three dietary intervention groups: a Mediterranean diet supplemented with extra-virgin olive oil, a Mediterranean diet supplemented with nuts, or a control diet. Enrollment began on June 25, 2003, and the last participant was recruited on June 30, 2009. The analyses in this report were based on a database locked as of September 2011 and included primary end-point events occurring through December 1, 2010. Randomization was concealed with the use of closed envelopes ${ }^{8}$ during part of the pilot phase of the study, but envelopes were not used for the remainder of the study. A computer-generated random-number sequence provided randomization tables for the 11 participating sites, which encompassed 169 clinics. These tables included four strata (men $<70$ years of age, men $\geq 70$ years of age, women $<70$ years of age, and women $\geq 70$ years of age) and were initially generated for 1000 participants (250 per stratum) for each site. We did not use blocks for randomization. Further details on the use of these tables at each of the 11 sites can 
be found on pages 8 and 9 and 78 through 82 in the Supplementary Appendix. All the sites were given the same randomization sequence.

There were departures from the randomization procedures that had been specified in the protocol that were not described in our original report. We included 425 participants who shared a household with a previously enrolled participant. These 425 participants were not randomly assigned but were assigned to the same intervention as the member of the household who was already enrolled (Tables S2 and S3 in the Supplementary Appendix). This was done to allow the recruitment of eligible household members and to avoid members of the same household being assigned to different diets. After the study had begun, the steering committee approved this protocol change. The protocol was not amended, and this protocol change was not described in the original report published in the Journal. In July 2017, we learned that at 1 of the 11 study sites (Site D), 467 participants were not randomly assigned as individual participants but instead were assigned according to clinic - that is, all the participants in each clinic received the same intervention ( 2 clinics assigned a Mediterranean diet with extra-virgin olive oil, 5 assigned a Mediterranean diet with nuts, and 4 assigned a control diet) (see pages 9 and 10 in the Supplementary Appendix for additional details). In addition, review of the documentation about randomization procedures and of the actual assignments to the three groups suggested that the randomization tables were inconsistently used in another study site (Site B, 593 participants) (see pages 10 and 11 in the Supplementary Appendix for details).

\section{INTERVENTIONS AND MEASUREMENTS}

The dietary intervention ${ }^{8,13-15}$ is detailed on pages 20 through 24 in the Supplementary Appendix. The specific recommended diets are summarized in Table 1. Participants in the group assigned to a Mediterranean diet with extra-virgin olive oil received 1 liter of the oil per week per household, with the recommendation to consume at least 4 tablespoons per day of extra-virgin olive oil per person. Participants in the group assigned to a Mediterranean diet with nuts received $30 \mathrm{~g}$ of mixed nuts per day per person (15 $\mathrm{g}$ of walnuts, $7.5 \mathrm{~g}$ of hazelnuts, and $7.5 \mathrm{~g}$ of almonds) at no cost, and those in the control group received small nonfood gifts. No total calorie restriction was advised, nor was physical activity promoted.
Table 1. Summary of Dietary Recommendations to Participants in the Mediterranean-Diet Groups and the Control-Diet Group.

\begin{tabular}{|c|c|}
\hline Food & Goal \\
\hline \multicolumn{2}{|l|}{ Mediterranean diet } \\
\hline \multicolumn{2}{|l|}{ Recommended } \\
\hline Olive oil* & $\geq 4$ tbsp/day \\
\hline Tree nuts and peanuts $†$ & $\geq 3$ servings/wk \\
\hline Fresh fruits & $\geq 3$ servings/day \\
\hline Vegetables & $\geq 2$ servings/day \\
\hline Fish (especially fatty fish), seafood & $\geq 3$ servings/wk \\
\hline Legumes & $\geq 3$ servings/wk \\
\hline Sofritoł & $\geq 2$ servings/wk \\
\hline White meat & Instead of red meat \\
\hline Wine with meals (optionally, only for habitual drinkers) & $\geq 7$ glasses $/ w k$ \\
\hline \multicolumn{2}{|l|}{ Discouraged } \\
\hline Soda drinks & $<1$ drink/day \\
\hline Commercial bakery goods, sweets, and pastries $\mathbb{}$ & $<2$ servings/wk \\
\hline Spread fats & $<1$ serving/day \\
\hline Red and processed meats & $<$ l serving/day \\
\hline \multicolumn{2}{|l|}{ Low-fat diet (control) q } \\
\hline \multicolumn{2}{|l|}{ Recommended } \\
\hline Low-fat dairy products & $\geq 3$ servings/day \\
\hline Bread, potatoes, pasta, rice & $\geq 3$ servings/day \\
\hline Fresh fruits & $\geq 3$ servings/day \\
\hline Vegetables & $\geq 2$ servings/day \\
\hline Lean fish and seafood & $\geq 3$ servings/wk \\
\hline \multicolumn{2}{|l|}{ Discouraged } \\
\hline Vegetable oils (including olive oil) & $\leq 2 \mathrm{tbsp} /$ day \\
\hline Commercial bakery goods, sweets, and pastries $\mathbb{3}$ & $\leq l$ serving/wk \\
\hline Nuts and fried snacks & $\leq l$ serving/wk \\
\hline Red and processed fatty meats & $\leq l$ serving/wk \\
\hline Visible fat in meats and soups $\|$ & Always remove \\
\hline Fatty fish, seafood canned in oil & $\leq l$ serving/wk \\
\hline Spread fats & $\leq l$ serving/wk \\
\hline Sofritotr & $\leq 2$ servings/wk \\
\hline
\end{tabular}

* The amount of olive oil includes oil used for cooking and salads and oil consumed in meals eaten outside the home. In the group assigned to the Mediterranean diet with extra-virgin olive oil, the goal was to consume $50 \mathrm{~g}$ (approximately 4 tbsp) or more per day of the polyphenol-rich olive oil supplied, instead of the ordinary refined variety, which is poor in polyphenols. The participants received a free supply ( 15 liters every 3 months) to include the oil used for cooking and family needs.

$\uparrow$ For participants assigned to the Mediterranean diet with nuts, the recommended consumption was one daily serving $(30 \mathrm{~g}$, composed of $15 \mathrm{~g}$ of walnuts, $7.5 \mathrm{~g}$ of almonds, and $7.5 \mathrm{~g}$ of hazelnuts). Participants received for free the needed allotments of tree nuts in packages of $2 \mathrm{~kg}$ of walnuts, $1 \mathrm{~kg}$ of almonds, and $1 \mathrm{~kg}$ of hazelnuts every 3 months, with the extra amounts to be shared with family members.

$†$ Sofrito is a sauce made with tomato and onion, often including garlic and aromatic herbs, and slowly simmered with olive oil.

$\int$ Commercial bakery goods, sweets, and pastries (not homemade) included cakes, cookies, biscuits, and custard.

9 Up to September 2006, a brief personal recommendation and a leaflet with written guidelines to attain these goals (see page 53 in the Supplementary Appendix) were given to participants on a yearly basis. Starting in October 2006, the intensity of these recommendations was increased, including also group sessions and personal advice repeated every 3 months (i.e., with the same intensity and frequency of contacts as in the two Mediterranean-diet groups). The composition of the recommended diet, however, was not changed.

\| Participants were advised to remove the visible fat (or the skin) of chicken, duck, pork, lamb, or veal before cooking and the fat of soups, broths, and cooked meat dishes before consumption. 
For participants in the two Mediterraneandiet groups, dietitians held individual and group dietary-training sessions at the baseline visit and quarterly thereafter. In each session, participants completed a 14-item dietary questionnaire to assess adherence to the Mediterranean diet ${ }^{8,16}$ (Table S4 in the Supplementary Appendix) so that personalized advice could be provided to the study participants in these groups. Questionnaire scores ranged from 0 to 14 , with scores lower than 10 defined as low adherence to the Mediterranean diet.

Participants in the control group also received dietary training at the baseline visit and completed the 14-item questionnaire at baseline to assess their adherence to the Mediterranean diet. During the first 3 years of the study, they received a leaflet explaining the low-fat diet (see page 53 in the Supplementary Appendix) on a yearly basis. However, the realization that the more infrequent visit schedule and less intense support for the control group might be limitations of the study prompted us to amend the protocol in October 2006. Thereafter, participants who were assigned to the control diet received personalized advice and were invited to group sessions with the same frequency and intensity as those in the Mediterranean-diet groups, with the use of a separate 9-item dietary questionnaire (Table S5 in the Supplementary Appendix). Scores ranged from 0 to 9 , with higher scores indicating greater adherence to a low-fat diet. Except for the Site $\mathrm{D}$ clinics discussed above and 11 clinics at Site I, all clinics of sufficient size delivered all three of the interventions (see page 11 in the Supplementary Appendix).

A general medical questionnaire, a 137-item validated food-frequency questionnaire, ${ }^{17}$ and the Minnesota Leisure-Time Physical Activity Questionnaire were administered on a yearly basis. ${ }^{13}$ Information from the food-frequency questionnaire was used to estimate intake of energy and nutrients. Weight, height, and waist circumference were directly measured annually. ${ }^{18}$ Biomarkers of adherence, including urinary hydroxytyrosol levels (to confirm adherence in the group receiving extravirgin olive oil) and plasma alpha-linolenic acid levels (to confirm adherence in the group receiving mixed nuts), were measured in random subsamples of participants at 1,3 , and 5 years (Figs. S9 and S10 in the Supplementary Appendix).

\section{END POINTS}

The primary end point was a composite of myocardial infarction, stroke, and death from cardiovascular causes. Secondary end points were stroke, myocardial infarction, death from cardiovascular causes, and death from any cause. We used four sources of information to identify end points: repeated contacts with participants, contacts with family physicians, a yearly review of medical records, and consultation of the $\mathrm{Na}$ tional Death Index. All medical records that were related to end points were examined by the end-point adjudication committee, whose members were unaware of the intervention-group assignments. Only end points that were confirmed by the adjudication committee and that occurred between June 25, 2003, and December 1, 2010, were included in the analyses. The criteria for adjudicating primary and secondary end points are detailed on pages 26 and 27 in the Supplementary Appendix.

\section{STATISTICAL ANALYSIS}

We initially estimated that a sample of 9000 participants would be required to provide a statistical power of $80 \%$ to detect a $20 \%$ lower risk of the primary end point in each Mediterraneandiet group than in the control-diet group during a 4-year follow-up period, assuming an absolute cumulative risk of $12 \%$ in the control group..$^{13,19}$ In April 2008, on the advice of the data and safety monitoring board and on the basis of lower-than-expected rates of end-point events, the sample size was recalculated as 7400 participants, with the assumption of a 6-year follow-up period because of slower-than-expected recruitment and an underlying absolute cumulative risk of the primary end point of $8.8 \%$ in the control group and 6.6\% in the Mediterranean-diet groups. The relationships between enrollment size and statistical power, under several assumptions, are shown in Figure S6 in the Supplementary Appendix.

Yearly interim analyses began on March 2008 after a median of 2 years of follow-up. With the use of O'Brien-Fleming stopping boundaries, the $P$ values for stopping the study at each yearly interim analysis were $5 \times 10^{-6}, 0.001,0.009$, and 0.02 for benefit and $9 \times 10^{-5}, 0.005,0.02$, and 0.05 for adverse effects. ${ }^{20}$ The stopping boundary for the benefit of the Mediterranean diets with re- 
spect to the primary end point was crossed at the fourth interim evaluation; on July 22, 2011, the data and safety monitoring board recommended stopping the study on the basis of end points documented through December 1, 2010. After the study was stopped, we advised all the participants, including those in the control group, to follow the Mediterranean diet.

The interim and original primary analyses estimated differences between the groups assigned to different interventions (intention-to-treat analyses). The information on protocol deviations was not considered in these analyses. Participants were followed from the baseline visit until the occurrence of a primary end-point event, death, or the last contact date from either medical records or study visits. We did not record the date of randomization and thus do not report the time between randomization and the baseline visit; for all the participants, we used the date of the baseline visit as time 0 in our analyses. No participant had a primary or secondary end-point event between randomization and baseline according to our review of the medical records.

We constructed Kaplan-Meier cumulative-incidence curves according to intervention group and calculated hazard ratios on an intention-to-treat basis, with the control group as the reference, using a Cox model with indicators for the Mediterranean diet with extra-virgin olive oil and the Mediterranean diet with nuts. We used robust variance estimators to account for intracluster correlations in all Cox models, considering as clusters the members of the same household and the participants in the same clinic of Site D. We compared baseline characteristics across the three groups and conducted analyses that did not rely on the assumption that all the participants were randomly assigned and that randomization would distribute baseline characteristics of the participants equally across intervention groups. Our main analysis was a multivariable model stratified according to site, sex, and educational level (five categories); to account for potential imbalances in baseline risk factors among the intervention groups, the model included nine other baseline variables as covariates (see page 12 in the Supplementary Appendix). This model was also adjusted for propensity scores that used 30 baseline variables to estimate the probability of assignment to each of the intervention groups (detailed on pages 12 through 17 in the Supplementary Appendix).

Prespecified subgroup analyses were conducted according to sex, age, body-mass index (BMI), status with respect to cardiovascular risk factors, and baseline adherence to the Mediterranean diet. In sensitivity analyses, we excluded the 1588 participants whose randomization procedures were known or suspected to have deviated from the protocol: all 652 participants from Site D ( 35 were second members of a household), 593 participants from Site B (47 were second members of a household), and another $343 \mathrm{sec}-$ ond household members from other sites. In addition, we performed sensitivity analyses to assess how strong and prevalent an unmeasured confounder would have to be to explain the observed results (Table S25 in the Supplementary Appendix). We also adjusted for missing data and loss to follow-up, implemented other exclusions, and used alternative analytic approaches (see pages 30 through 35 and Figs. S2 and S4 in the Supplementary Appendix).

A secondary analysis estimated the per-protocol effect ${ }^{21}$ of the Mediterranean diet as compared with the control diet that would have been observed if all the participants had adhered to their assigned interventions throughout the follow-up period. For participants assigned to the Mediterranean-diet groups, adherence was defined as a score of 10 or higher on the 14-item questionnaire. For those assigned to the low-fat diet, adherence was defined as a fat intake of $30 \%$ or less of total energy intake according to the foodfrequency questionnaires that were administered annually to the three groups or a score or 6 or higher on the 9-item questionnaire. We censored data for participants when they first stopped adhering to their assigned intervention, estimated inverse-probability weights to adjust for postrandomization prognostic factors, and estimated the hazard ratio for an end-point event in the Mediterranean-diet groups as compared with the low-fat diet group. ${ }^{22,23}$

The validity of the per-protocol effect estimate relies on several assumptions. It assumes that loss to follow-up, data collection, and adherence can be treated as sequentially randomized at each time point, given the measured prognostic factors before and after randomization..$^{22}$ Both Mediterranean-diet groups were combined for 
precision because only $39 \%$ of the events remained uncensored after the application of our strict definition of adherence. We used the predicted values from this model after adding a product term between intervention and time to estimate cumulative-incidence curves (see pages 36 through 38 in the Supplementary Appendix for details).

\section{RESULTS}

\section{BASELINE CHARACTERISTICS}

From June 25, 2003, through June 30, 2009, a total of 8713 candidates were screened for eligibility, and 7447 were assigned to one of the three intervention groups (Fig. S7 in the Supplementary Appendix). Their baseline characteristics according to intervention group are shown in Table 2, and in Table S23 in the Supplementary Appendix. The exclusion of participants whose randomization procedures were known to have deviated from the protocol did not materially change these results. Drug-treatment regimens at baseline were similar for participants in the three groups, and they continued to be balanced during the follow-up period (Table S6 in the Supplementary Appendix).

Participants were followed for a median of 4.8 years (interquartile range, 2.8 to 5.8 ). After the baseline visit, 210 participants $(2.8 \%)$ chose not to attend subsequent visits $(1.2 \%$ of the participants assigned to a Mediterranean diet with extra-virgin olive oil, $2.7 \%$ of those assigned to a Mediterranean diet with nuts, and $4.7 \%$ of those in the control group). The rate of study discontinuation ( $>2$ years since last contact) was $11.3 \%$ in the control group and $4.9 \%$ in the Mediterranean-diet groups; subsequent follow-up was based on reviews of medical records (Fig. S7 and Table S24 in the Supplementary Appendix). Participants who dropped out of the study were, on average, 1.4 years younger than those who remained in the study and had a higher BMI (the weight in kilograms divided by the square of height in meters) by 0.4 , a higher waist-to-height ratio (by 0.01 ), and a lower score for adherence to the Mediterranean diet (by 1.0 points on the 14-item questionnaire) at baseline.

\section{ADHERENCE TO THE DIETARY INTERVENTION}

The scores on the 14-item Mediterranean-diet questionnaire increased over the follow-up period for the participants in the two Mediterraneandiet groups (Table S7 and Fig. S8 in the Supplementary Appendix). There were substantial differences between the Mediterranean-diet groups and the control group in 12 of the 14 items (Table S7 in the Supplementary Appendix). Changes in biomarkers also indicated good adherence to the dietary assignments (Figs. S9 and S10 in the Supplementary Appendix).

Participants in the two Mediterranean-diet groups increased weekly servings of fish (by 0.3 servings) and legumes (by 0.4 servings) in comparison with those in the control group (Table S8 in the Supplementary Appendix). In addition, participants assigned to a Mediterranean diet with extra-virgin olive oil and those assigned to a Mediterranean diet with nuts increased their consumption of extra-virgin olive oil (to 50 and $32 \mathrm{~g}$ per day, respectively) and nuts (to 0.9 and 6 servings per week, respectively). The main nutrient changes in the Mediterranean-diet groups reflected the fat content and composition of the supplemental foods (Tables S9 and S10 in the Supplementary Appendix). No relevant diet-related adverse effects were reported (see page 38 in the Supplementary Appendix). We found little difference in changes in physical activity among the three groups.

\section{END POINTS}

In the intention-to-treat analysis, there were 96 primary end-point events in the group assigned to a Mediterranean diet with extra-virgin olive oil (3.8\%), 83 in the group assigned to a Mediterranean diet with nuts (3.4\%), and 109 in the control group $(4.4 \%)$. The respective incidence rates were 8.1, 8.0, and 11.2 per 1000 personyears, and the 5-year absolute risks were $3.6 \%$, $4.0 \%$, and $5.7 \%$, respectively (Table 3 ). The unadjusted hazard ratios that used robust variance estimators to account for intracluster correlations were 0.70 (95\% confidence interval [CI], 0.53 to 0.92) for a Mediterranean diet with extra-virgin olive oil and 0.70 (95\% CI, 0.53 to 0.94 ) for a Mediterranean diet with nuts as compared with the control diet.

Results of our primary analyses that included adjustment for propensity scores and 12 baseline participant characteristics were similar to those of the unadjusted analyses, with hazard ratios of 0.69 (95\% CI, 0.53 to 0.91 ) for a Mediterranean diet with extra-virgin olive oil and 0.72 (95\% CI, 
Table 2. Baseline Characteristics of the Participants, According to Intervention Group.*

\begin{tabular}{|c|c|c|c|}
\hline Characteristic & $\begin{array}{l}\text { Mediterranean } \\
\text { Diet with EVOO } \\
(N=2543)\end{array}$ & $\begin{array}{l}\text { Mediterranean } \\
\text { Diet with Nuts } \\
(\mathrm{N}=2454)\end{array}$ & $\begin{array}{l}\text { Control Diet } \\
(N=2450)\end{array}$ \\
\hline Female sex - no. (\%) $\dagger$ & $1493(58.7)$ & $1326(54.0)$ & $1463(59.7)$ \\
\hline Age - yri & $67.0 \pm 6.2$ & $66.7 \pm 6.1$ & $67.3 \pm 6.3$ \\
\hline \multicolumn{4}{|l|}{ Race or ethnic group — no. (\%) $\nleftarrow$} \\
\hline White, from Europe & $2470(97.1)$ & $2390(97.4)$ & $2375(96.9)$ \\
\hline Hispanic, from Central or South America & $35(1.4)$ & $29(1.2)$ & $38(1.6)$ \\
\hline Other & $38(1.5)$ & $35(1.4)$ & $37(1.5)$ \\
\hline \multicolumn{4}{|l|}{ Smoking status — no. (\%) } \\
\hline Never smoked & $1572(61.8)$ & $1465(59.7)$ & $1527(62.3)$ \\
\hline Former smoker & $618(24.3)$ & $634(25.8)$ & $584(23.8)$ \\
\hline Current smoker & $353(13.9)$ & $355(14.5)$ & $339(13.8)$ \\
\hline Body-mass indextð & $29.9 \pm 3.7$ & $29.7 \pm 3.8$ & $30.2 \pm 4.0$ \\
\hline Waist circumference $-\mathrm{cm}$ & $100 \pm 10$ & $100 \pm 10$ & $101 \pm 11$ \\
\hline Waist-to-height ratio -9 & $0.63 \pm 0.06$ & $0.63 \pm 0.06$ & $0.63 \pm 0.07$ \\
\hline Hypertension — no. (\%)\| & $2088(82.1)$ & $2024(82.5)$ & $2050(83.7)$ \\
\hline Type 2 diabetes - no. (\%) †*** & $1282(50.4)$ & $1143(46.6)$ & $1189(48.5)$ \\
\hline Dyslipidemia - no. (\%) †† & $1821(71.6)$ & $1799(73.3)$ & $1763(72.0)$ \\
\hline Family history of premature CHD - no. (\%) & $576(22.7)$ & $532(21.7)$ & $560(22.9)$ \\
\hline \multicolumn{4}{|l|}{ Medication use - no. (\%) } \\
\hline ACE inhibitors & $1236(48.6)$ & $1223(49.8)$ & $1216(49.6)$ \\
\hline Diureticst' & $534(21.0)$ & $477(19.4)$ & $562(22.9)$ \\
\hline Other antihypertensive agents & $725(28.5)$ & $710(28.9)$ & $758(30.9)$ \\
\hline Statins & $1039(40.9)$ & $964(39.3)$ & $983(40.1)$ \\
\hline Other lipid-lowering agents & $121(4.8)$ & $145(5.9)$ & $126(5.1)$ \\
\hline Insulin & $124(4.9)$ & $126(5.1)$ & $134(5.5)$ \\
\hline Oral hypoglycemic agents $†$ & $768(30.2)$ & $680(27.7)$ & $757(30.9)$ \\
\hline Antiplatelet therapy & $475(18.7)$ & $490(20.0)$ & $513(20.9)$ \\
\hline Hormone-replacement therapy $\iint$ & $42(2.8)$ & $35(2.6)$ & $39(2.7)$ \\
\hline
\end{tabular}

* Plus-minus values are means \pm SD. Percentages may not total 100 because of rounding. ACE denotes angiotensinconverting enzyme, and EVOO extra-virgin olive oil.

$\dagger \mathrm{P}<0.05$ for comparisons between groups.

$\neq$ Race and ethnic group were determined by the staff of the trial (nurses or dietitians).

$\int$ The body-mass index is the weight in kilograms divided by the square of the height in meters.

I The waist-to-height ratio (an index of central obesity) is the waist circumference divided by height.

|| Hypertension was defined as a systolic blood pressure of $140 \mathrm{~mm} \mathrm{Hg}$ or higher, a diastolic blood pressure of $90 \mathrm{~mm} \mathrm{Hg}$ or higher, or the use of antihypertensive therapy.

**: Diabetes was defined as a fasting blood glucose level of $126 \mathrm{mg}$ per deciliter $(7.0 \mathrm{mmol}$ per liter) or higher on two occasions, a 2-hour plasma glucose level of $200 \mathrm{mg}$ per deciliter (11.1 mmol per liter) or higher during a 75-g oral glucose-tolerance test, or the use of antidiabetic medication.

$\dagger$ ' Dyslipidemia was defined as a low-density lipoprotein cholesterol level higher than $160 \mathrm{mg}$ per deciliter $(4.1 \mathrm{mmol}$ per liter), a high-density lipoprotein cholesterol level of $40 \mathrm{mg}$ per deciliter $(1.0 \mathrm{mmol}$ per liter) or lower in men or $50 \mathrm{mg}$ per deciliter (1.3 mmol per liter) or lower in women, or the use of lipid-lowering therapy.

A family history of premature coronary heart disease (CHD) was defined as a diagnosis of the disease in a male firstdegree relative younger than 55 years of age or in a female first-degree relative younger than 65 years of age.

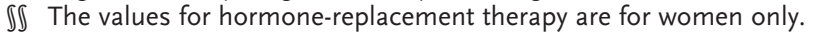


Table 3. Estimates of Cardiovascular Events, According to Intervention Group.*

Mediterranean

End Point

No. of person-yr of follow-up

Primary end point $†$

No. of events

Incidence rate per 1000 person-yr $(95 \% \mathrm{Cl})$

5 -yr absolute risk — \% $(95 \% \mathrm{Cl}) \doteqdot$

Secondary end points

Stroke

No. of events

Incidence rate per 1000 person-yr $(95 \% \mathrm{Cl})$

5 -yr absolute risk — \% $(95 \% \mathrm{Cl})$

Myocardial infarction

No. of events

Incidence rate per 1000 person-yr $(95 \% \mathrm{Cl})$

5 -yr absolute risk — \% $(95 \% \mathrm{Cl})$

Death from cardiovascular causes

No. of events

Incidence rate per 1000 person-yr (95\% Cl)

5 -yr absolute risk — \% $(95 \% \mathrm{Cl})$

Death from any cause

No. of events

Incidence rate per 1000 person-yr $(95 \% \mathrm{Cl})$

5 -yr absolute risk — \% $(95 \% \mathrm{Cl})$

ITT analysis: hazard ratio for each Mediterranean diet vs. control $(95 \% \mathrm{Cl}) \sqrt{\text { d }}$

Primary end point

Unadjusted

Adjusted 9

Secondary end points $₫$

Stroke

Myocardial infarction

Death from cardiovascular causes

Death from any cause

ITT analysis: hazard ratio for Mediterranean diets combined vs. control $(95 \% \mathrm{CI}) \mathbb{\int}$

Primary end point

Unadjusted

Adjusted

Secondary end points 9

Stroke

Myocardial infarction

Death from cardiovascular causes

Death from any cause
Diet with EVOO

( $N=2543)$

11852

96

$8.1(6.6-9.9)$

$3.6(2.8-4.5)$

49

$4.1(3.1-5.5)$

$1.7(1.3-2.4)$

37

$3.1(2.2-4.3)$

$1.4(1.0-2.1)$

26

$2.2(1.4-3.2)$

$1.0(0.6-1.5)$

118

$10.0(8.2-11.9)$

$4.4(3.6-5.4)$

32

58

$3.1(2.1-4.4)$

$5.9(4.5-7.7)$

$1.5(1.1-2.3)$

$3.0(2.3-3.9)$

31

$3.0(2.0-4.2)$

$1.6(1.1-2.3)$

38

$3.9(2.8-5.3)$

$2.1(1.5-2.9)$

31

30

$3.0(2.0-4.2)$

$3.1(2.1-4.4)$

$1.4(0.9-2.1)$

$1.6(1.1-2.3)$

114

116

$11.7(9.6-14.0)$

$11.2(9.3-13.4)$

$5.4(4.4-6.7)$

$5.4(4.4-6.6)$
Diet with Nuts Control Diet

9763

109

$1.2(9.2-13.5)$

$5.7(4.6-6.9)$

$\begin{array}{lll}0.70(0.53-0.92) & 0.70(0.53-0.94) & 1.00 \text { (ref) } \\ 0.69(0.53-0.91) & 0.72(0.54-0.95) & 1.00 \text { (ref) } \\ 0.65(0.44-0.95) & 0.54(0.35-0.82) & 1.00 \text { (ref) } \\ 0.82(0.52-1.30) & 0.76(0.47-1.25) & 1.00 \text { (ref) } \\ 0.62(0.36-1.06) & 1.02(0.63-1.67) & 1.00(\text { ref) } \\ 0.90(0.69-1.18) & 1.12(0.86-1.47) & 1.00(\text { ref) }\end{array}$




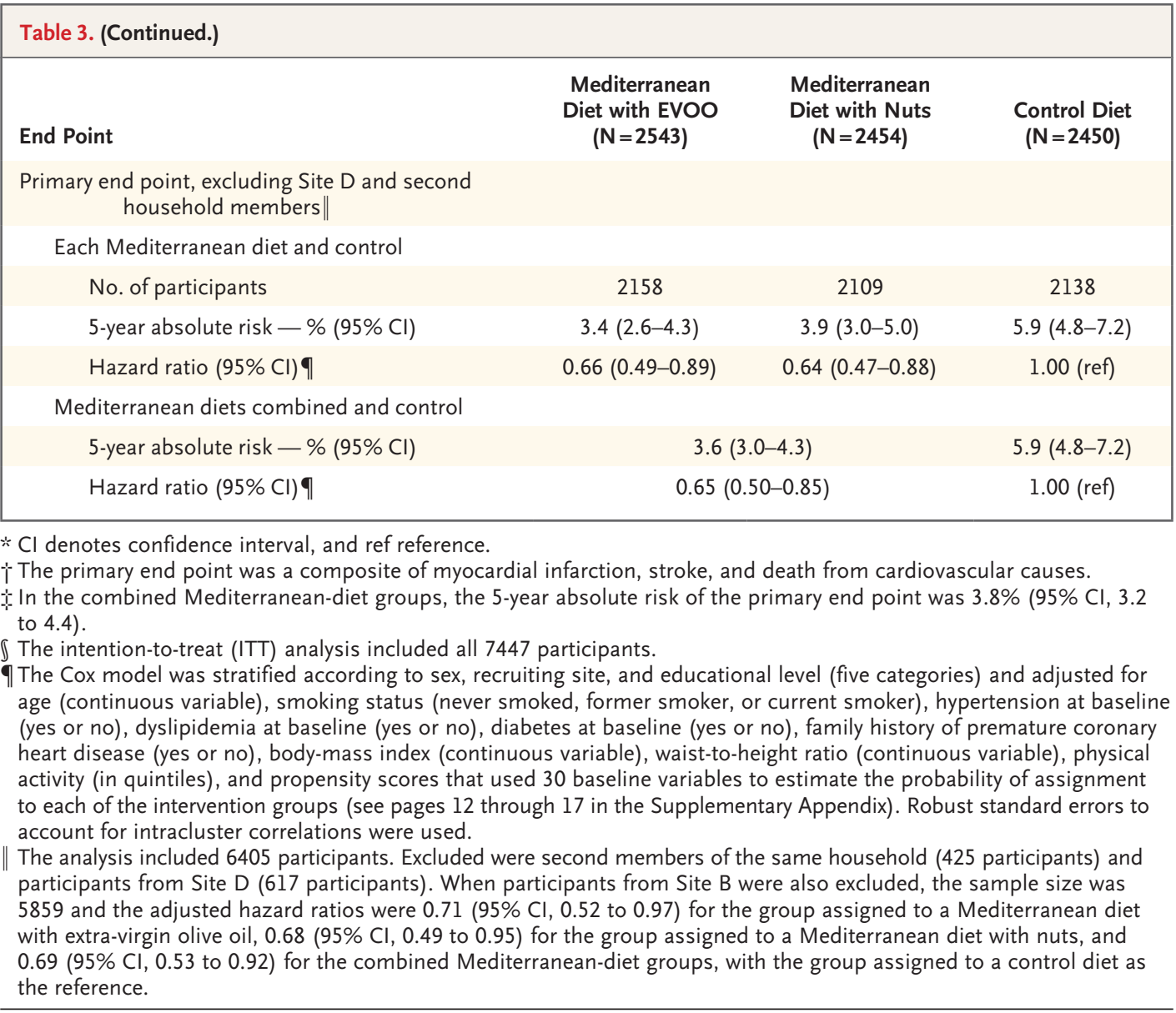

0.54 to 0.95 ) for a Mediterranean diet with nuts (Fig. 1A and Table 3). There were similar results on three alternative analyses: one that was adjusted with inverse-probability weighting (models 3A through 3C in Fig. S2 in the Supplementary Appendix), one that included adjustments for the Framingham risk score ${ }^{24}$ (models 6A through 6C in Fig. S2 in the Supplementary Appendix), and one that omitted participants known or suspected to have been assigned to an intervention group without individual randomization (Table 3 and Figs. 2 and 3, and Figs. S2 and S4 in the Supplementary Appendix). The results for secondary end points are shown in Table 3 and Figure 1B.

To provide an alternative, noncausal explanation of the observed association (i.e., to change the point estimate of the hazard ratio to $\geq 1.0$ ), an unmeasured binary confounder would need to be present in at least $40 \%$ of the control group but in less than $25 \%$ of each Mediterranean-diet group and be associated with a relative risk of more than 4 for the primary end point. For further details, see Table S25 in the Supplementary Appendix.

To explore the effect of the October 2006 protocol change that was implemented for the control group to intensify nutritional counseling, we conducted separate analyses of the participants recruited before the protocol change and those recruited after the protocol change. The hazard ratios for the Mediterranean diet (both groups merged) as compared with the control diet were 0.77 (95\% CI, 0.59 to 1.00) for the participants recruited before October 2006 and 0.49 (95\% CI, 0.26 to 0.92 ) for those recruited in October 2006 or later $(\mathrm{P}=0.21$ for heterogeneity).

The per-protocol (adherence-adjusted) hazard ratio for the primary end point was 0.42 (95\% CI, 0.24 to 0.63 ) for the Mediterranean diet as compared with the control diet (Fig. 3); the estimated absolute differences in incidence between the combined Mediterranean-diet groups and 


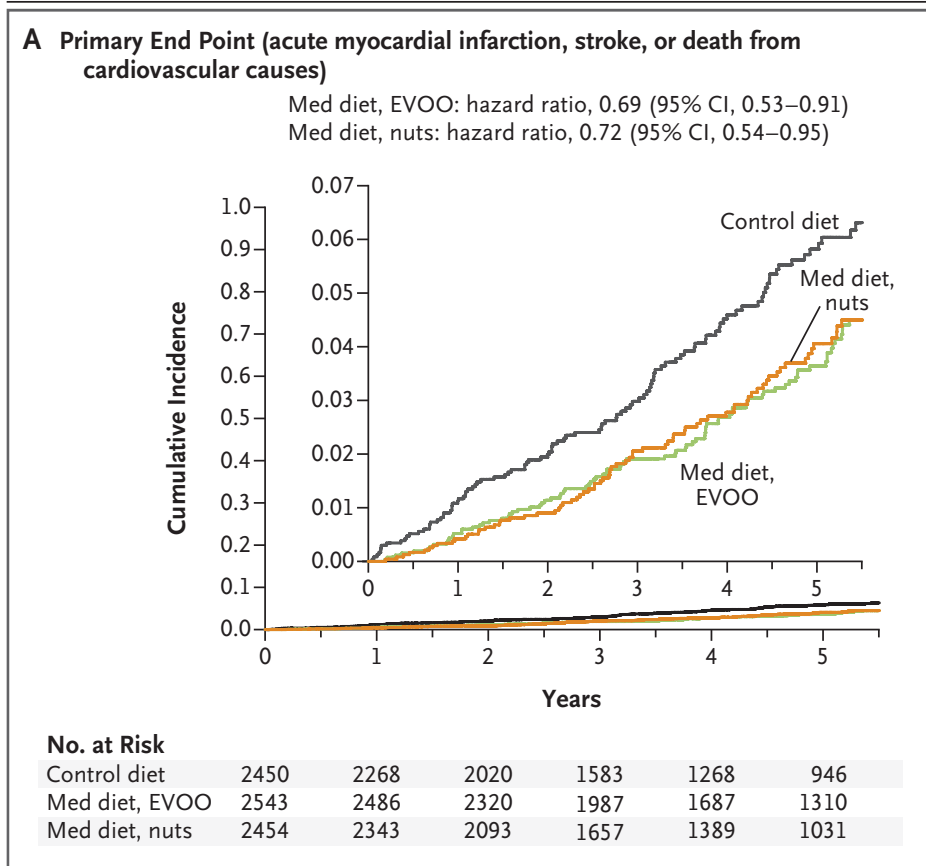

B Total Mortality

Med diet, EVOO: hazard ratio, 0.90 (95\% Cl, 0.69-1.18)

Med diet, nuts: hazard ratio, $1.12(95 \% \mathrm{Cl}, 0.86-1.47)$

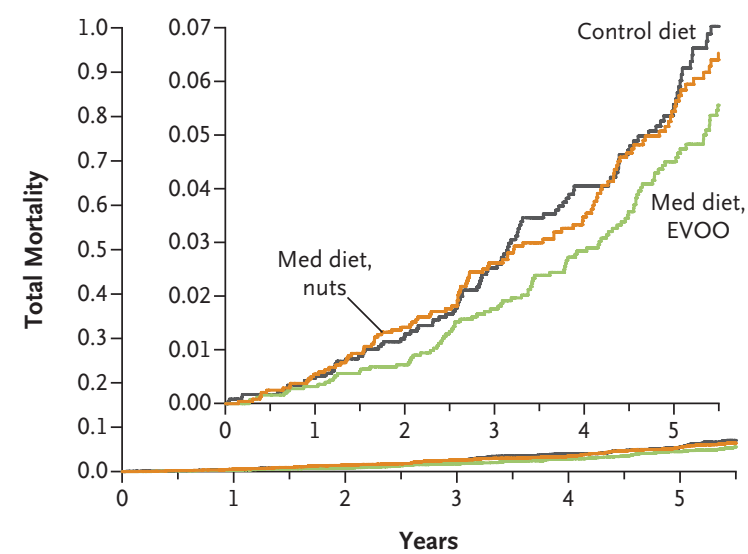

No. at Risk

Control diet

Med diet, nuts

2543
2454

$$
\begin{aligned}
& 2270 \\
& 2486
\end{aligned}
$$$$
2345
$$

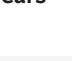

$$
2324
$$$$
2097
$$

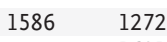

1991

1662
1272

1395
949

1310

1037

the control group were $0.67,1.38$, and 2.00 percentage points at 12, 24, and 36 months after enrollment, respectively (see pages 36 through 38 in the Supplementary Appendix). The results of additional sensitivity and subgroup analyses were also consistent with the results of our primary analyses (Figs. 2 and 3, and Figs. S2, S4, and S12 in the Supplementary Appendix).

Figure 1. Kaplan-Meier Estimates of the Cumulative Incidence of End-Point Events in the Total Study Population.

Panel A shows the incidence of the primary end point (a composite of acute myocardial infarction, stroke, and death from cardiovascular causes), and Panel B shows total mortality. The insets show the same data on an expanded y axis. Hazard ratios were stratified according to sex, recruiting site, and educational level (five categories) and adjusted for age (continuous variable), smoking (never smoked, former smoker, or current smoker), hypertension (yes or no), dyslipidemia (yes or no), diabetes (yes or no), family history of premature coronary heart disease, body-mass index (continuous variable), waist-to-height ratio (continuous variable), physical activity (in quintiles), and propensity scores that estimated the probability of assignment to each intervention group on the basis of 30 baseline variables (see pages 12 through 17 in the Supplementary Appendix). Robust standard errors to account for intracluster correlations were used. $\mathrm{Cl}$ denotes confidence interval, EVOO extra-virgin olive oil, and Med Mediterranean.

\section{DISCUSSION}

In this study involving high-risk persons without cardiovascular disease, assignment to an energyunrestricted Mediterranean diet supplemented with either extra-virgin olive oil or nuts was associated with a lower risk of major cardiovascular events over a period of 5 years than assignment to a control (low-fat) diet, with a relative difference of $30 \%$ and an absolute difference of 1.7 to 2.1 percentage points. Our analysis, which incorporated information about adherence to the diets, suggests that the difference in rates of cardiovascular events between those assigned to the Mediterranean diets and those assigned the control diet was greater among participants with better adherence. These results support previously reported benefits of the Mediterranean diet for cardiovascular risk reduction from a randomized trial. ${ }^{4,25,26}$ Our findings are also consistent with those of previous observational studies. ${ }^{2,5,23,25-33}$ Table S11 in the Supplementary Appendix summarizes the findings from systematic reviews on this issue.

In response to a 2017 report $^{11}$ suggesting that distributions of baseline variables in the PREDIMED trial were significantly different from what would have been expected to result from randomization, we conducted an extensive review 


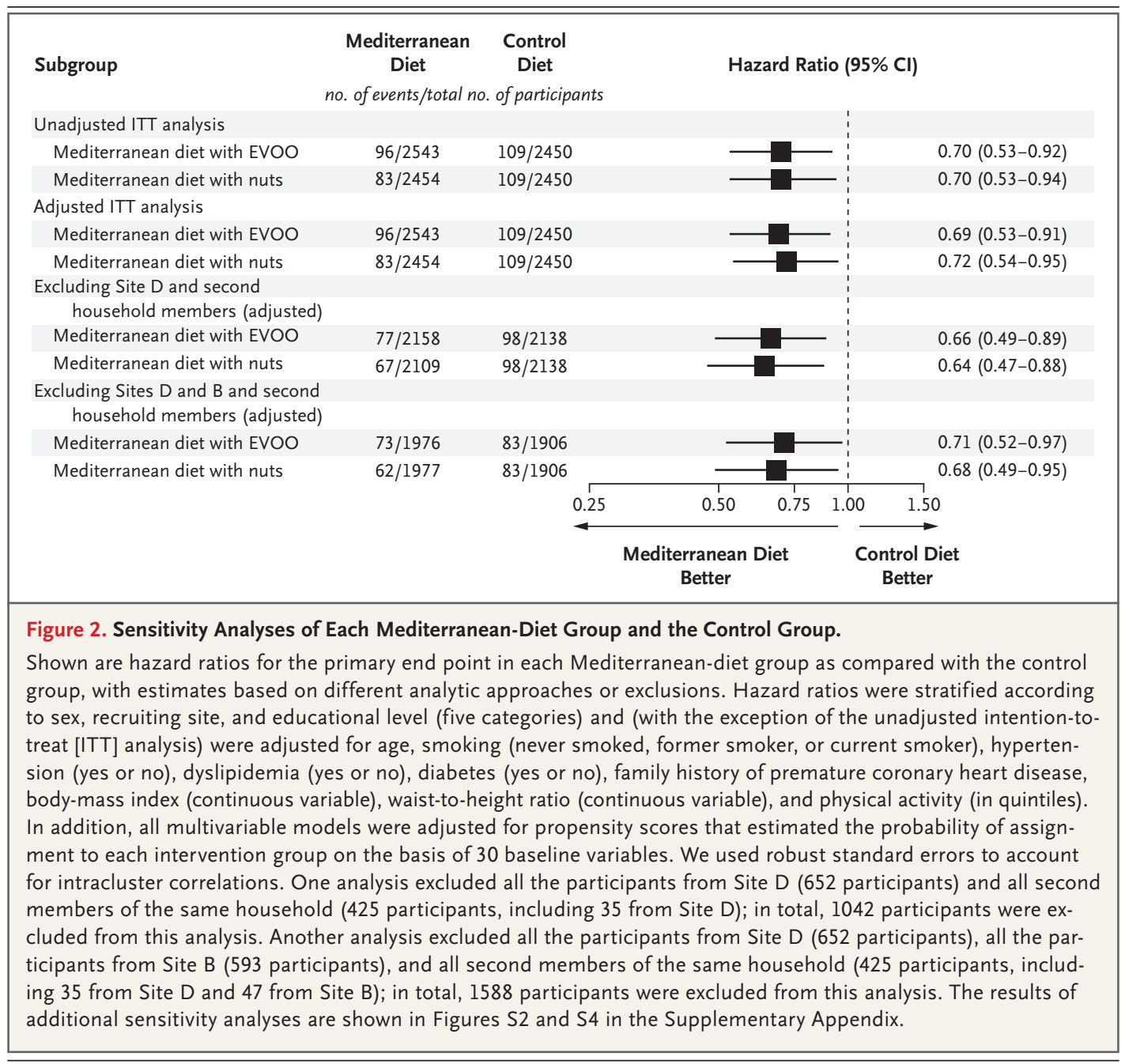

of the documentation and data at the 11 recruitment sites. After sharing this information with the editors of the Journal, we withdrew our original report of this trial and now publish this new report. Despite some departures from the randomization protocol, most of the baseline covariates were balanced across groups, and there was no meaningful difference in the predicted risks of future cardiovascular events across the three groups (Fig. S13 in the Supplementary Appendix).

We reanalyzed the data using methods that do not rely exclusively on the assumption that all the participants had been randomly assigned to intervention groups and that adjusted for baseline characteristics and propensity scores estimating probabilities of assignment to each intervention on the basis of 30 baseline covariates. The results of our reanalyses (Figs. 2 and 3 and Table 3, and Figs. S2 and S4 in the Supplementary Appendix) were similar to the results that we originally reported. In addition, reanalyses of our data did not reveal any evidence that certain lifestyle or treatment factors that are potentially related to the risk of cardiovascular disease either biased the results or might provide an alternative explanation for the observed benefits of the Mediterranean-diet interventions on cardiovascular disease. Analyses that excluded participants whose assignment to an intervention group was known or suspected not to have followed the randomization protocol (participants from Sites D and $\mathrm{B}$ and second household members) yielded results consistent with the results of our primary analysis.

The retention rate was higher in the group 


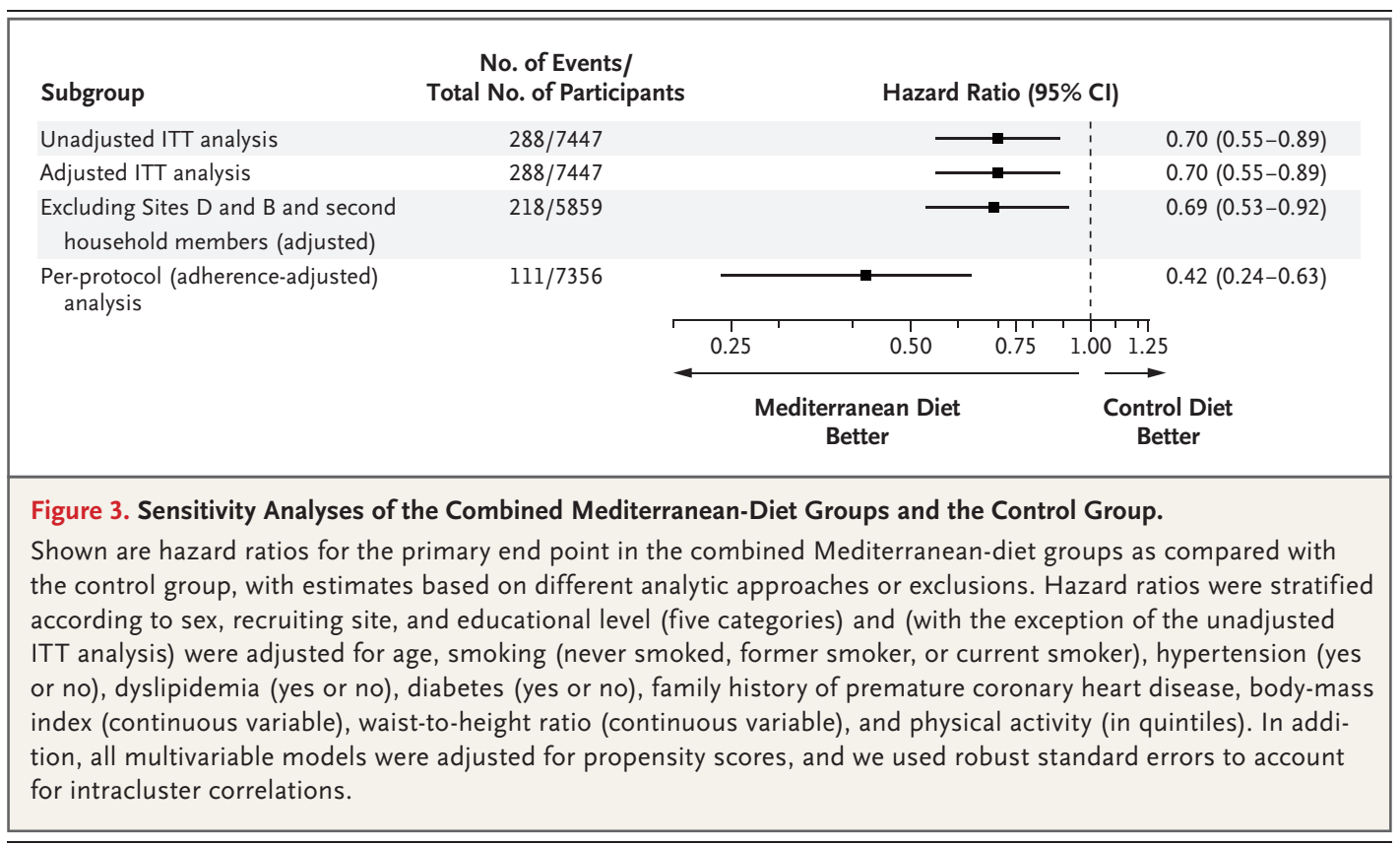

assigned to a Mediterranean diet with extra-virgin olive oil than in the other two groups (the group assigned to a Mediterranean diet with nuts and the control group). These two groups were also slightly smaller in size, which resulted in a larger number of person-years of follow-up in the group assigned to a Mediterranean diet with extra-virgin olive oil. The different follow-up had the potential to bias the incidence rates toward lower rates in the group assigned to a Mediterranean diet with extra-virgin olive oil. However, analyses that used multiple imputation and inverse-probability weighting to adjust for a potential selection bias due to differential losses to follow-up yielded estimates consistent with the main analysis (see pages 30 through 35 and Fig. S4 in the Supplementary Appendix). An additional limitation of our study is that participants were at high cardiovascular risk; whether the results can be generalized to persons at lower risk requires further research.

As with many clinical trials, the observed rates of cardiovascular events were lower than anticipated, with reduced statistical power to separately assess components of the primary end point. However, favorable trends were seen for both stroke and myocardial infarction. It is possible, but not likely, that some cardiovascular events were not detected (see pages 28 and 29 in the Supplementary Appendix).
Even though participants in the control group received advice to reduce fat intake, changes in total fat on the food-frequency questionnaire were small and the largest differences at the end of the study were in the distribution of fat subtypes. The interventions were intended to improve the overall dietary pattern, but the major between-group differences involved the supplemental items, extra-virgin olive oil and nuts. Differences were also observed in the consumption of fish and legumes but not in the consumption of other food groups. (It is worth noting that on the 14-item Mediterranean-diet questionnaire, there were substantial between-group differences in 12 of the 14 items.) The modest betweengroup differences according to the food-frequency questionnaire can be explained by the facts that most study participants had been consuming a baseline diet similar to the study Mediterranean diet and that the control group was given recommendations for a healthy diet, factors that raise the question of how applicable our results may be to high-risk persons in other countries. Answering this question will require further research. ${ }^{3}$

In conclusion, in this primary prevention study involving persons at high risk for cardiovascular events, those assigned to an energy-unrestricted Mediterranean diet, supplemented with extravirgin olive oil or nuts, had a lower rate of major cardiovascular events than those assigned to a 
reduced-fat diet. Our findings support a beneficial effect of the Mediterranean diet for the primary prevention of cardiovascular disease.

Supported by Instituto de Salud Carlos III, Spanish Ministry of Health, through grants provided to research networks specifically developed for the study (RTIC G03/140, to Dr. Estruch, and RTIC RD 06/0045, to Dr. Martínez-González); Centro de Investigación Biomédica en Red de Fisiopatología de la Obesidad y Nutrición; and grants from Centro Nacional de Investigaciones Cardiovasculares (CNIC 06/2007), Fondo de Investigación Sanitaria-Fondo Europeo de Desarrollo Regional (PI04-2239, PI 05/2584, CP06/00100, PI07/0240, PI07/1138, PI07/0954, PI 07/0473, PI10/01407, PI10/02658, PI11/01647, and P11/02505), Ministerio de Ciencia e Innovación (AGL-2009-13906-C02 and AGL2010-22319-C03), Fundación
Mapfre 2010, Consejería de Salud de la Junta de Andalucía (PI0105/2007), Public Health Division of the Department of Health of the Autonomous Government of Catalonia, Generalitat Valenciana (ACOMP06109, GVACOMP2010-181, GVACOMP2011-151, CS2010-AP-111, and CS2011-AP-042), and Regional Government of Navarra (P27/2011). Dr. Hernán was supported by a grant (ME-1503-28119) from the Patient-Centered Outcomes Research Institute.

Disclosure forms provided by the authors are available with the full text of this article at NEJM.org.

We thank the participants for their involvement in the study; and Joan Vila from Institut Hospital del Mar d'Investigacions Mèdiques, Barcelona, and Estefanía Toledo from the University of Navarra, Pamplona, for their assistance with statistical analyses.

\section{APPENDIX}

The authors' full names and academic degrees are as follows: Ramón Estruch, M.D., Ph.D., Emilio Ros, M.D., Ph.D., Jordi Salas-Salvadó, M.D., Ph.D., Maria-Isabel Covas, D.Pharm., Ph.D., Dolores Corella, D.Pharm., Ph.D., Fernando Arós, M.D., Ph.D., Enrique Gómez-Gracia, M.D., Ph.D., Valentina Ruiz-Gutiérrez, Ph.D., Miquel Fiol, M.D., Ph.D., José Lapetra, M.D., Ph.D., Rosa M. LamuelaRaventos, D.Pharm., Ph.D., Lluís Serra-Majem, M.D., Ph.D., Xavier Pintó, M.D., Ph.D., Josep Basora, M.D., Ph.D., Miguel A. Muñoz, M.D., Ph.D., José V. Sorlí, M.D., Ph.D., J. Alfredo Martínez, D.Pharm., M.D., Ph.D., Montserrat Fitó, M.D., Ph.D., Alfredo Gea, D.Pharm., Ph.D., Miguel A. Hernán, M.D., Dr.P.H., and Miguel A. Martínez-González, M.D., Ph.D.

The authors' affiliations are as follows: the PREDIMED (Prevención con Dieta Mediterránea) Network (RD 06/0045) (R.E., J.S.-S., F.A., E.G.-G., V.R.-G., R.M.L.-R., L.S.-M., X.P., J.B., J.V.S., J.A.M., M.A.M.-G.) and Centro de Investigación Biomédica en Red de Fisiopatología de la Obesidad y Nutrición (R.E., E.R., J.S.-S., M.-I.C., D.C., F.A., M. Fiol, J.L., R.M.L.-R., L.S.-M., X.P., J.B., J.V.S., J.A.M., M. Fitó, A.G., M.A.M.-G.), Instituto de Salud Carlos III, Madrid, the Department of Internal Medicine, Hospital Clinic (R.E.), the Lipid Clinic, Department of Endocrinology and Nutrition, Institut d'Investigacions Biomèdiques August Pi i Sunyer (E.R.), and the Department of Nutrition, Food Sciences, and Gastronomy, Faculty of Pharmacy and Food Sciences, Xarxa de Referència en Tecnologia dels Aliments, Instituto de Investigación en Nutrición y Seguridad Alimentaria (R.M.L.-R.), University of Barcelona, Institut Hospital del Mar d'Investigacions Mèdiques (M.-I.C., M. Fitó), the Lipids and Vascular Risk Unit, Internal Medicine, Hospital Universitario de Bellvitge, Hospitalet de Llobregat (X.P.), and the Primary Care Division, Catalan Institute of Health, Institut Universitari d'Investigació en Atenció Primària (IDIAP)-Jordi Gol (M.A.M.), Barcelona, the Human Nutrition Department, Hospital Universitari Sant Joan, Institut d'Investigació Sanitària Pere Virgili, Universitat Rovira i Virgili, Reus (J.S.-S.), the Department of Preventive Medicine, University of Valencia (D.C., J.V.S.), and the Primary Care Division, Valencia Institute of Health (J.V.S.), Valencia, the Department of Cardiology, University Hospital of Álava, Vitoria (F.A.), the Department of Preventive Medicine, University of Malaga, Malaga (E.G.-G.), Instituto de la Grasa, Consejo Superior de Investigaciones Científicas (V.R.-G.), and the Department of Family Medicine, Primary Care Division of Sevilla, San Pablo Health Center (J.L.), Seville, the Institute of Health Sciences, University of the Balearic Islands, and Hospital Son Espases, Palma de Mallorca (M. Fiol), the Research Institute of Biomedical and Health Sciences, Instituto Universitario de Investigaciones Biomédicas y Sanitarias, University of Las Palmas de Gran Canaria, Las Palmas (L.S.-M.), the Primary Care Division, Catalan Institute of Health, IDIAP-Jordi Gol, Tarragona-Reus (J.B.), and the Departments of Nutrition and Food Sciences and Physiology (J.A.M.) and Preventive Medicine and Public Health (A.G., M.A.M.-G.), University of Navarra, Pamplona - all in Spain; and the Departments of Epidemiology and Biostatistics (M.A.H.) and Nutrition (M.A.M.-G.), Harvard T.H. Chan School of Public Health, and the Harvard-MIT Division of Health Sciences and Technology (M.A.H.), Boston.

\section{REFERENCES}

1. Willett WC, Sacks F, Trichopoulou A, et al. Mediterranean diet pyramid: a cultural model for healthy eating. Am J Clin Nutr 1995;61:Suppl:1402S-1406S.

2. Sofi F, Abbate R, Gensini GF, Casini A. Accruing evidence on benefits of adherence to the Mediterranean diet on health: an updated systematic review and metaanalysis. Am J Clin Nutr 2010;92:118996.

3. Martínez-González MÁ, Hershey MS, Zazpe I, Trichopoulou A. Transferability of the Mediterranean diet to non-Mediterranean countries: what is and what is not the Mediterranean diet. Nutrients 2017; 9(11):E1226.

4. de Lorgeril M, Salen P, Martin JL, Monjaud I, Delaye J, Mamelle N. Mediterranean diet, traditional risk factors, and the rate of cardiovascular complications after myocardial infarction: final report of the Lyon Diet Heart Study. Circulation 1999;99:779-85.

5. Mente A, de Koning L, Shannon HS, Anand SS. A systematic review of the evidence supporting a causal link between dietary factors and coronary heart disease. Arch Intern Med 2009;169:65969.

6. Esposito K, Marfella R, Ciotola M, et al. Effect of a Mediterranean-style diet on endothelial dysfunction and markers of vascular inflammation in the metabolic syndrome: a randomized trial. JAMA 2004; 292:1440-6.

7. Vincent-Baudry S, Defoort C, Gerber $\mathrm{M}$, et al. The Medi-RIVAGE study: reduction of cardiovascular disease risk factors after a 3-mo intervention with a Mediterranean-type diet or a low-fat diet. Am J Clin Nutr 2005;82:964-71.

8. Estruch R, Martínez-González MA,
Corella D, et al. Effects of a Mediterranean-style diet on cardiovascular risk factors: a randomized trial. Ann Intern Med 2006;145:1-11.

9. Shai I, Schwarzfuchs D, Henkin Y, et al. Weight loss with a low-carbohydrate, Mediterranean, or low-fat diet. N Engl J Med 2008;359:229-41.

10. Estruch R, Ros E, Salas-Salvadó J, et al. Primary prevention of cardiovascular disease with a Mediterranean diet. N Engl J Med 2013;368:1279-90.

11. Carlisle JB. Data fabrication and other reasons for non-random sampling in 5087 randomised, controlled trials in anaesthetic and general medical journals. Anaesthesia 2017;72:944-52.

12. Estruch R, Ros E, Salas-Salvadó J, et al. Retraction and republication: Primary prevention of cardiovascular disease with a Mediterranean diet. N Engl J Med 2013; 
368:1279-90. N Engl J Med. DOI: 10.1056/ NEJMc1806491.

13. Martínez-González MA, Corella D, Salas-Salvadó J, et al. Cohort profile: design and methods of the PREDIMED study. Int J Epidemiol 2012;41:377-85.

14. PREDIMED home page (http://www .predimed.es/).

15. Zazpe I, Sanchez-Tainta A, Estruch R, et al. A large randomized individual and group intervention conducted by registered dietitians increased adherence to Mediterranean-type diets: the PREDIMED study. J Am Diet Assoc 2008;108:1134-44. 16. Schröder H, Fitó M, Estruch R, et al. A short screener is valid for assessing Mediterranean diet adherence among older Spanish men and women. J Nutr 2011; 141:1140-5.

17. Fernández-Ballart JD, Piñol JL, Zazpe I, et al. Relative validity of a semi-quantitative food-frequency questionnaire in an elderly Mediterranean population of Spain. Br J Nutr 2010;103:1808-16.

18. Salas-Salvadó J, Fernández-Ballart J, Ros E, et al. Effect of a Mediterranean diet supplemented with nuts on metabolic syndrome status: one-year results of the PREDIMED randomized trial. Arch Intern Med 2008;168:2449-58.

19. ALLHAT Officers and Coordinators for the ALLHAT Collaborative Research Group. Major outcomes in moderately hypercholesterolemic, hypertensive patients randomized to pravastatin vs usual care: the Antihypertensive and Lipid-Lowering Treatment to Prevent Heart Attack Trial (ALLHAT-LLT). JAMA 2002;288:2998-3007. 20. O'Brien PC, Fleming TR. A multiple testing procedure for clinical trials. Biometrics 1979;35:549-56.

21. Hernán MA, Hernández-Díaz S, Robins JM. Randomized trials analyzed as observational studies. Ann Intern Med 2013; 159:560-2.

22. Hernán MA, Robins JM. Per-protocol analyses of pragmatic trials. $\mathrm{N}$ Engl J Med 2017;377:1391-8.

23. Toh S, Hernández-Díaz S, Logan R, Robins JM, Hernán MA. Estimating absolute risks in the presence of nonadherence: an application to a follow-up study with baseline randomization. Epidemiology 2010;21:528-39.

24. D'Agostino RB Sr, Vasan RS, Pencina MJ, et al. General cardiovascular risk profile for use in primary care: the Framing ham Heart Study. Circulation 2008;117: 743-53.

25. Kris-Etherton P, Eckel RH, Howard BV, St. Jeor S, Bazzarre TL. AHA science advisory: Lyon Diet Heart Study — benefits of a Mediterranean-style, National Cholestero Education Program/American Heart Association Step I dietary pattern on cardiovascular disease. Circulation 2001;103: 1823-5.

26. de Lorgeril M, Renaud S, Mamelle N, et al. Mediterranean alpha-linolenic acidrich diet in secondary prevention of coronary heart disease. Lancet 1994;343:1454-9. 27. Bendinelli B, Masala G, Saieva C, et al. Fruit, vegetables, and olive oil and risk of coronary heart disease in Italian women: the EPICOR Study. Am J Clin Nutr 2011; 93:275-83.

28. Samieri C, Féart C, Proust-Lima C, et al. Olive oil consumption, plasma oleic acid, and stroke incidence: the Three-City Study. Neurology 2011;77:418-25.

29. Buckland G, Travier N, Barricarte A, et al. Olive oil intake and CHD in the European Prospective Investigation into Cancer and Nutrition Spanish cohort. Br J Nutr 2012;108:2075-82

30. Kris-Etherton PM, Hu FB, Ros E, Sabaté $J$. The role of tree nuts and peanuts in the prevention of coronary heart disease: multiple potential mechanisms. J Nutr 2008;138:1746S-1751S.

31. Ros E, Tapsell LC, Sabaté J. Nuts and berries for heart health. Curr Atheroscler Rep 2010;12:397-406.

32. Trichopoulou A, Bamia C, Trichopoulos D. Anatomy of health effects of Mediterranean diet: Greek EPIC prospective cohort study. BMJ 2009;338:b2337.

33. Buckland G, Mayén AL, Agudo A, et al. Olive oil intake and mortality within the Spanish population (EPIC-Spain). Am J Clin Nutr 2012;96:142-9.

Copyright (c) 2018 Massachusetts Medical Society. 